\title{
miR-24 may be a negative regulator of menin in lung cancer
}

\author{
YUNHU PAN ${ }^{1 *}$, HONGMEI WANG $^{2 *}$, DEBIN MA $^{3 *}$, ZHIYU JI $^{1}$, LIMIN LUO $^{1}$, FANGYU CAO $^{1}$, \\ FANGFANG HUANG ${ }^{1}$, YANGYANG LIU ${ }^{4}$, YUSHU DONG ${ }^{4}$ and YITAN CHEN ${ }^{1}$ \\ ${ }^{1}$ Department of Respiratory Medicine, No. 92 Hospital of PLA, Nanping, Fujian 341000; \\ ${ }^{2}$ Department of Hematology, No. 463 Hospital of PLA, Shenyang, Liaoning 110840; \\ ${ }^{3}$ Department of Respiratory Medicine; ${ }^{4}$ Institute of Neuroscience, \\ General Hospital of Shenyang Military Area Command, \\ Shenyang, Liaoning 110840, P.R. China
}

Received June 29, 2017; Accepted March 7, 2018

DOI: $10.3892 /$ or.2018.6327

\begin{abstract}
The incidence of lung cancer in China increases annually, and effective targets for the diagnosis and treatment of lung cancer are urgently needed. miRNAs are currently considered to be involved in the regulation of tumor development and growth. miR-24 has been found to contribute to the development of several tumors. Menin is a key tumor suppressor gene, and its expression is generally low in lung cancer. The effects of miR-24 on the biological behavior of lung cancer cells were detected by MTT and Transwell assays. In the present study, miR-24 was found to be associated with menin, affecting the activity of the SMAD3 pathway in lung cancer by inhibiting menin expression. miR-24 may promote the growth and metastasis and inhibit the apoptosis of lung cancer cells by targeting menin. Therefore, the aim of the present study was to provide a new theoretical basis for the targeted therapy of lung cancer.
\end{abstract}

\section{Introduction}

According to WHO statistics, the incidence of lung cancer in China increases annually by $25 \%(1,2)$. Mutations of key proto-oncogenes, such as K-ras, ALK and EGFR, and tumor-suppressor genes, such as p53, are important molecular mechanisms underlying the occurrence of lung cancer (3-7).

Correspondence to: Dr Yitan Chen, Department of Respiratory Medicine, No. 92 Hospital of PLA, Nanping, Fujian 341000, P.R. China

E-mail: zhangguoxin2016@126.com

Dr Yushu Dong, Institute of Neuroscience, General Hospital of Shenyang Military Area Command, Shenyang, Liaoning 110840, P.R. China

E-mail: dongyushu463@163.com

${ }^{*}$ Contributed equally

Key words: miR-24, menin, lung cancer, SMAD3
In recent years, miRNAs have been attracting increasing attention in the research of tumor pathogenesis, (8-10) and their sensitivity and specificity for the diagnosis of lung cancer have been found to be relatively high $(11,12)$.

Men1, a critical tumor-suppressor gene in multiple endocrine neoplasia type 1 , encodes a recently identified protein, menin, of which the emerging roles in cancer development have been attracting increasing attention (13). It has also been reported that menin plays a role in suppressing hyperplasia or tumor development in several other organs, such as the lung, prostate and breast, and it exacerbates diabetes in mouse models (14). However, the molecular mechanisms underlying the role of menin in lung cancer remain unclear.

miR-24 is reportedly able to promote the development of several types of tumors. For example, miR-24 promoted the proliferation and inhibited the apoptosis of HeLa cells $(15,16)$, and has also been found to be highly expressed in Hodgkin's lymphoma (17-21). However, the association between miR-24 and menin has not yet been fully elucidated.

In the present study, the RNA expression of miR-24 and menin in lung cancer and adjacent tissues were observed, and it was demonstrated that miR-24 also promotes cell growth and metastasis by targeting menin.

\section{Materials and methods}

Tissue samples and cell lines. A total of 70 samples of tumor tissues and adjacent tissues ( $\geq 2 \mathrm{~cm}$ from the tumor) from patients with lung cancer who were diagnosed and treated at the General Hospital of Shenyang Military Command were obtained between May 2013 and June 2015. The study protocols were approved by the Ethics Committee of the General Hospital of Shenyang Military Command. Patient consent was obtained in writing according to institutional regulations.

A549 and NCI-H446 cells (obtained from the Wuhan Cell Bank) were maintained in Dulbecco's modified Eagle's medium (Gibco; Thermo Fisher Scientific, Waltham, MA, USA) supplemented with $10 \%$ fetal bovine serum (HyClone; GE Healthcare Life Sciences, Chicago, IL, USA), $1 \%$ penicillin/streptomycin, $1 \% \mathrm{~L}$-glutamine, under $5 \% \mathrm{CO}_{2}$ at $37^{\circ} \mathrm{C}$. 
Transfections. A549 and NCI-H 446 cells $\left(1 \times 10^{5}\right)$ were plated in 6-well plates and transfected with $100 \mathrm{nM}$ of miR-24 mimic (UGGCUCAGUUCAGCAGGAACAG)/ mimic or control (UCACAACCUCCUAGAAAGAGU AGA) and miR-24 inhibitor (CUGUUCCUGCUGAACUGA GCCA)/inhibitor or control (CAGUACUUUUGUGUAGUA CAA) (Guangzhou RiboBio Co., Ltd., Guangzhou, China) after $24 \mathrm{~h}$ by Lipofectamine 2000 (Invitrogen; Thermo Fisher Scientific) according to the manufacturer's protocol. Menin and menin-del were transfected into cells with Lipofectamine 2000, and the experiments were conducted after $24 \mathrm{~h}$.

MTT assays. Cells were seeded at a density of $1 \times 10^{3}$ cells/well in 96-well plates, and transfected with different miRNAs. The MTT assay was performed $24 \mathrm{~h}$ later, using a microplate reader (Bio-Rad Laboratories, Inc., Hercules, CA, USA) to evaluate cell proliferation. The optical densities of the samples were measured at $490 \mathrm{~nm}$.

Hoechst 33258 assay. After transfection for $24 \mathrm{~h}$, washing with PBS, and addition of $1 \mu \mathrm{l}(1 \mathrm{mg} / \mathrm{ml})$ Hoechst 33258 for $10 \mathrm{~min}$, the samples were examined using fluorescence microscopy.

Transwell assay. Modified Boyden chambers with polycarbonate Nuclepore ${ }^{\mathrm{TM}}$ membranes (Corning Inc., Corning, NY, USA) were used to perform metastasis assays according to the manufacturer's protocol. After $24 \mathrm{~h}$, the transfected cells were seeded in Transwell chambers in serum-free media with or without Matrigel coating, while medium containing $30 \%$ fetal bovine serum was placed in the lower well. After $24 \mathrm{~h}$, the cells were washed with PBS, fixed with methanol for $20 \mathrm{~min}$, stained with crystal violet dye for $10 \mathrm{~min}$ and then counted under a light microscope.

Reverse transcription-quantitative polymerase chain reaction (RT-qPCR). RNA was extracted from tissue samples or cells using TRIzol reagent (Invitrogen; Thermo Fisher Scientific, Inc.) according to the manufacturer's protocol. cDNA synthesis was performed with the High-Capacity cDNA synthesis kit (Takara Bio, Inc., Otsu, Japan). RT-qPCR analysis was performed on an Applied Biosystems 7500 Real-Time PCR System according to the manufacturer's instructions (33). The primer sequences are shown in Table I. All the reactions were performed as previously described (34).

Western blot analysis. Tissues and cells were scraped and lysed in RIPA buffer (Sigma-Aldrich; Merck KGAa, Darmstadt, Germany). To determine the levels of different proteins, $30 \mu \mathrm{g}$ of protein from each sample was subjected to $10 \%$ SDS-PAGE and transferred onto a nitrocellulose membrane (Corning Inc.). Target proteins were probed with specific antibodies against menin (1:1,000; cat. no. sc-374371), SMAD3 (1:1,000; cat. no. sc-4709), cyclin D1 (1:1,000; cat. no. sc-4074), Bax (1:1,000; cat. no. sc-4239), Bcl-2 (1:1,000; cat. no. sc-56015), MMP2 (1:1,000; cat. no. sc-13594) and GAPDH (1:5,000; cat. no. sc-365062) (all from Santa Cruz Biotechnology, Inc., Dallas, TX, USA).
Dual-luciferase reporter assay. Dual-luciferase activity assays were performed as previously described (35). The menin 3'-untranslated region (UTR) was PCR-amplified and cloned into the pMIR-REPORT ${ }^{\mathrm{TM}}$ vector (Ambion; Thermo Fisher Scientific). The sequences were as follows: Menin-wt forward, 5'-TGCACACAGACAACCTGATCT-3' and reverse, 5'-ACACCGGAGCTGTCCAATTT-3'; and menin-del forward, 5'-CTGCACACAGACAACCTGATCT-3' and reverse, 5'-GCCATGGGGTACCTTTCCAG-3'. A549 cells were co-transfected with menin-wt or -del reporter vector and control plasmid in miR-24 mimic and miR-24 AS (antisense). Luciferase activity was determined with the Dual-Luciferase Reporter Assay System (Promega Corp., Madison, WI, USA) after $36 \mathrm{~h}$ of transfection.

Statistical analysis. All experiments were repeated at least three times. A correlation analysis with the log-rank test was used to evaluate the differences in the levels of possible prognostic factors. Statistical significance was evaluated with the two-tailed Student's t-test comparing two groups of data. Asterisks indicate significant differences of experimental groups compared with the corresponding control condition. Statistical analysis was performed using GraphPad Prism software (GraphPad, Inc. Inc., La Jolla, CA, USA) and statistical significance was defined as $\mathrm{P}<0.05$.

\section{Results}

Association of miR-24 and menin in lung cancer. The results revealed that the expression of miR-24 in the tumor tissues was found to be higher compared with that in the adjacent tissues of 70 lung cancer patients as determined by qPCR (Fig. 1A). The results of the Kaplan-Meier analysis demonstrated that patients with high miR-24 expression had a significantly decreased overall survival (Fig. 1B). Next, we observed that miR-24 was closely associated with the development of lung cancer. There was a correlation between miR-24 and the size of the tumor (Table II). Biological software (miRDB) predicted that miR-24 may target menin. Based on that finding, we analyzed the expression of menin in cancer tissues and adjacent tissues in the patients by qPCR (Fig. 1C). The results revealed that the expression level of menin was lower in tumor tissues than in adjacent normal tissues. In the Kaplan-Meier analysis, we observed that patients with a high expression of menin survived longer (Fig. 1D). It was also observed that miR-24 and menin were negatively correlated in these patients (Fig. 1E). Using the miRDB software, we identified a binding site in the 3'-UTR region of menin for miR-24 (Fig. 1F). The luciferase reporter assay demonstrated that the activity of menin was significantly inhibited following co-transfection with miR-24. This inhibitory effect was eliminated when the 3'-UTR region of menin was mutated. Furthermore, transfection with the miR-24 antisense strand did not inhibit the activity of menin (Fig. 1G).

miR-24 promotes the proliferation of lung cancer cells. The miR-24 mimic/inhibitor was transfected into lung cancer cells, and the effect of miR-24 on proliferation was then evaluated by MTT assay (Fig. 2A and B). It was observed that the proliferation of lung cancer cells was stimulated when miR-24 was overexpressed. Menin, SMAD3 and cyclin D1 were assessed 
Table I. Primer sequences for the detection of RNA expression.

\begin{tabular}{lll}
\hline Name & \multicolumn{1}{c}{ Forward primer $\left(5^{\prime}->3^{\prime}\right)$} & Reverse primer $\left(5^{\prime}->3^{\prime}\right)$ \\
\hline miR-24 & GTCGTATCCAGTGCAGGGTCCGAGGTATTCGC & GGACTGTCTTGGCATCCATGTAG \\
U6 & CTCGGATACGACCTC & \\
Menin & GCACACAGACAACCTGATCTTTT & AACGCTTCACGAATTTGCGT \\
Cyclin D1 & CCGAGGAGCTGCTGCAAATGG & TCGGGAACGTTGGTAGGGAT \\
Bcl-2 & GGTGAACTGGGGGAGGATTG & GAAATCGTGCGGGGTCATTGCG \\
Bax & AGCTGAGCGAGTGTCTCAAG & GGCAGGCATGTTGACTTCAC \\
MMP2 & TGATCTTGACCAGAATACCATCG & GTCCAATGTCCAGCCCATGA \\
GAPDH & AACGACCCCTTCATTGAC & GGCTTGCGAGGGAAGAAGTT \\
\end{tabular}

Table II. The relationship between miR-24 and lung cancer.

\begin{tabular}{|c|c|c|c|c|c|c|}
\hline \multirow[b]{2}{*}{ Parameters } & \multirow[b]{2}{*}{ Description } & \multirow[b]{2}{*}{ No. of patients } & \multicolumn{2}{|c|}{ miR-24 expression } & \multirow[b]{2}{*}{$\chi^{2}$} & \multirow[b]{2}{*}{ P-value } \\
\hline & & & Low & High & & \\
\hline \multirow[t]{2}{*}{ Sex } & Male & 44 & 17 & 27 & 0.381 & 0.537 \\
\hline & Female & 26 & 12 & 14 & & \\
\hline \multirow[t]{2}{*}{ Age (years) } & $<40$ & 22 & 10 & 12 & 0.214 & 0.643 \\
\hline & $\geq 40$ & 48 & 19 & 29 & & \\
\hline \multirow[t]{2}{*}{ Type } & Non-small cell & 60 & 27 & 33 & 2.208 & 0.137 \\
\hline & Small cell & 10 & 2 & 8 & & \\
\hline \multirow[t]{2}{*}{ Tumor size (cm) } & $<5$ & 32 & 18 & 14 & 5.337 & $0.021^{\mathrm{a}}$ \\
\hline & $\geq 5$ & 38 & 11 & 27 & & \\
\hline \multirow[t]{2}{*}{ Depth of invasion (pT) } & $\mathrm{T} 1, \mathrm{~T} 2$ & 42 & 11 & 31 & 10.048 & $0.002^{\mathrm{a}}$ \\
\hline & $\mathrm{T} 3, \mathrm{~T} 4$ & 28 & 18 & 10 & & \\
\hline \multirow[t]{2}{*}{ Lymph node metastasis $(\mathrm{pN})$} & Yes & 42 & 13 & 29 & 4.749 & $0.029^{\mathrm{a}}$ \\
\hline & No & 28 & 16 & 12 & & \\
\hline \multirow[t]{2}{*}{ Distant metastasis (pM) } & Yes & 9 & 1 & 8 & 3.912 & $0.048^{\mathrm{a}}$ \\
\hline & No & 61 & 28 & 3 & & \\
\hline \multirow[t]{2}{*}{ TNM stage } & I-II & 37 & 21 & 16 & 7.599 & $0.006^{\mathrm{b}}$ \\
\hline & III-IV & 33 & 8 & 25 & & \\
\hline
\end{tabular}

${ }^{\text {aP }}<0.05$ There is a difference in miR-24 between two groups. ${ }^{\mathrm{b}} \mathrm{P}<0.05$ There is a significant difference in miR-24 between two groups.

when miR-24 was overexpressed in A549 cells (Fig. 2C and D). The results demonstrated that miR-24 inhibited the expression of menin, and it also upregulated SMAD3 and cyclin D1 expression. In turn, when miR-24 was inhibited in A549 cells, menin expression was increased (Fig. 2E and F), whereas SMAD3 and cyclin D1 expression levels in A549 cells were decreased.

miR-24 inhibits the apoptosis of lung cancer cells. Since miR-24 affects menin to regulate the proliferation of lung cancer cells, it was hypothesized that the apoptosis of lung cancer cells may also be affected by targeting menin. miR-24 mimics/controls or miR-24 inhibitors/controls were transfected into A549 cells to observe the effect of miR-24 on the apoptosis of A549 cells (Fig. 3A and B). Hoechst 33258 staining revealed that miR-24 significantly inhibited the apoptosis of A549 cells. Next, we examined the protein and mRNA levels of menin, SMAD3, Bcl-2 and Bax in A549 cells overexpressing miR-24 (Fig. 3C and D). The results demonstrated that Bcl-2 expression was increased, whereas Bax expression was inhibited when miR-24 was overexpressed. It was then observed that the expression of Bcl-2 was inhibited and that of Bax was promoted at both the protein and mRNA levels after miR-24 inhibitor was transfected into A549 cells (Fig. 3E and F).

miR-24 promotes the metastasis of lung cancer cells. Transwell assays (with or without Matrigel) were used to investigate 
A
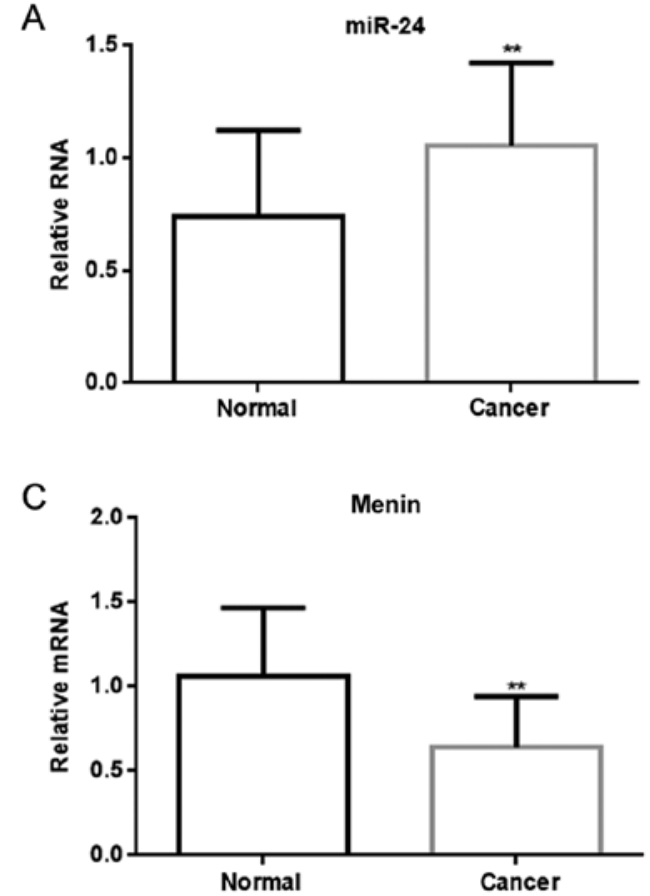

$E$

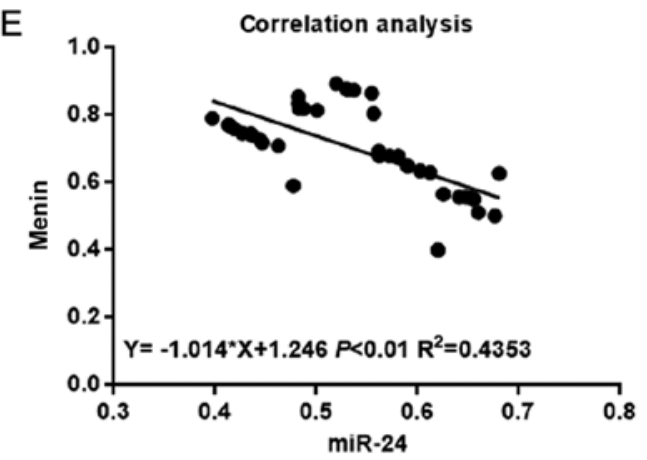

B

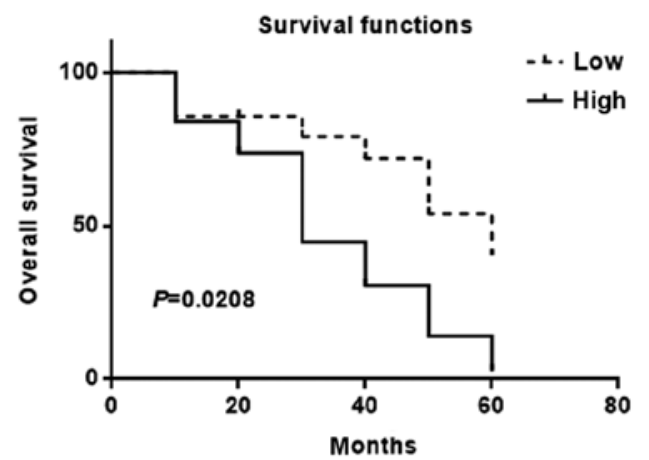

D

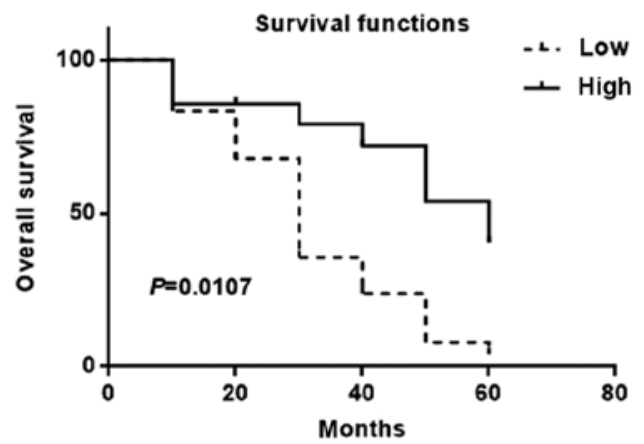

$\mathrm{F}$

g g a ua ucuca

Menin UTR 5' cucc gu ccu cugagcuga ucagu u

miR-24 gagg ca gga gacuugacu gguca u

a a c - c cacau

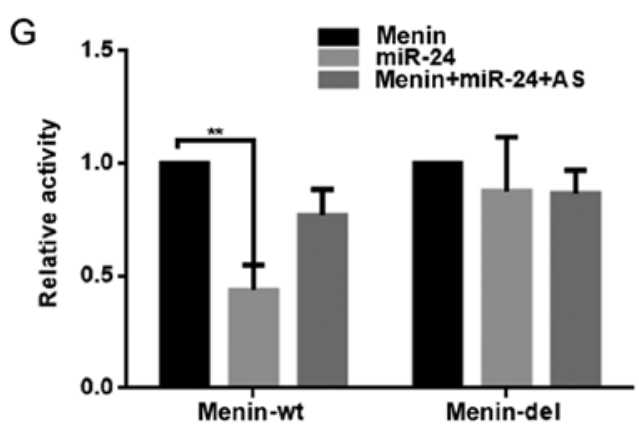

Figure 1. Association of miR-24 and menin in lung cancer. (A) The levels of miR-24 in 70 samples of lung cancer tissues and adjacent tissues were detected by quantitative polymerase chain reaction (qPCR) analysis. The expression of miR-24 was higher in lung cancer tissues. Data are shown as the mean \pm standard error of the mean (SEM). ${ }^{* *} \mathrm{P}<0.01$ vs. adjacent tissues. (B) Association between the expression of miR-24 and patient survival. The survival time of patients with high expression of miR-24 was shorter compared with that of patients with low expression of miR-24. (C) The levels of menin in 70 samples of lung cancer tissues and adjacent tissues were detected by qPCR. Data are shown as the mean $\pm \mathrm{SEM}$. ${ }^{* *} \mathrm{P}<0.01$ vs. adjacent tissues. (D) Association between the expression of menin and patient survival. The survival time of patients with high expression of menin was longer compared with that of patients with low expression of menin. (E) Correlation between the expression of miR-24 and menin. The expression of miR-24 was negatively correlated with the expression of menin. (F) miRDB predicted that miR-24 could specifically combine with menin. (G) The interaction between miR-24 and the menin 3'-UTR was assessed by luciferase reporter assays. Assays were performed by co-transfection of miR-24 or miR-24 antisense and menin-wt or menin-del. Data are shown as the mean \pm SEM. ${ }^{* *} \mathrm{P}<0.01$ vs. the menin group.

whether miR-24 is involved in the metastasis of lung cancer cells (Fig. 4A-D). A miR-24 mimic or miR-24 inhibitor was transfected into A549 and NCI-H446 cells. Subsequently, the Transwell results demonstrated that the migration and inva- sion of A549 and NCI-H446 cells were significantly enhanced when miR-24 was overexpressed; however, the migration and invasion of A549 and NCI-H446 cells were inhibited when miR-24 expression was decreased. Furthermore, the protein 
A

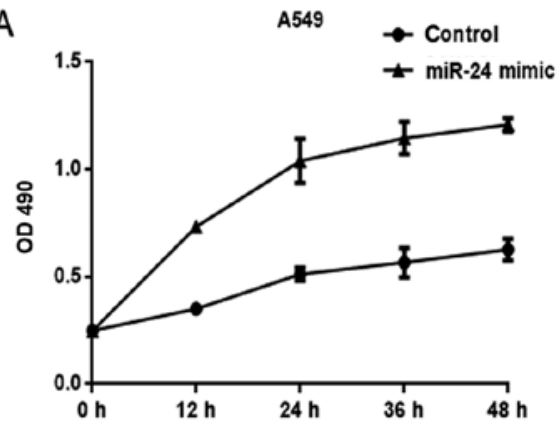

B

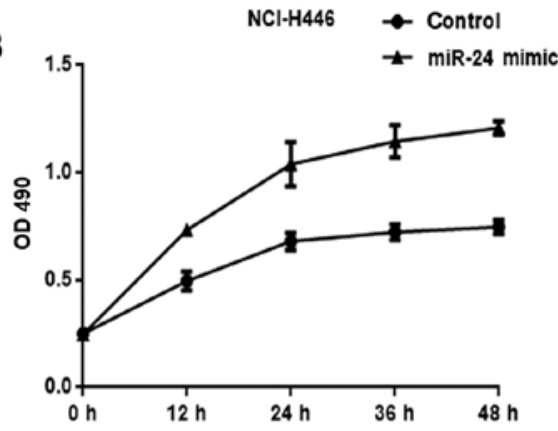

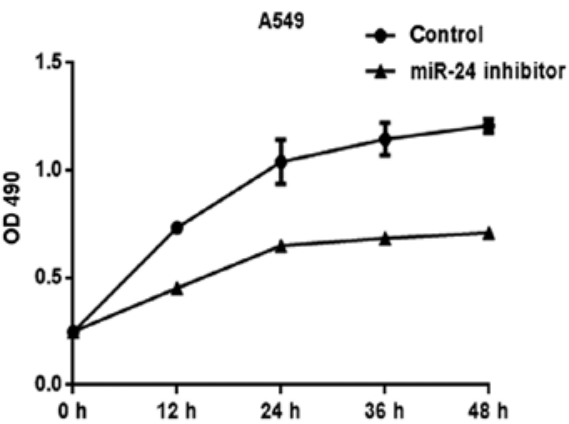

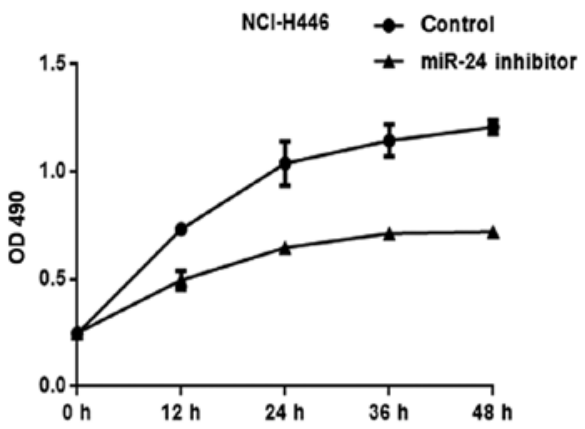

C con miR-24
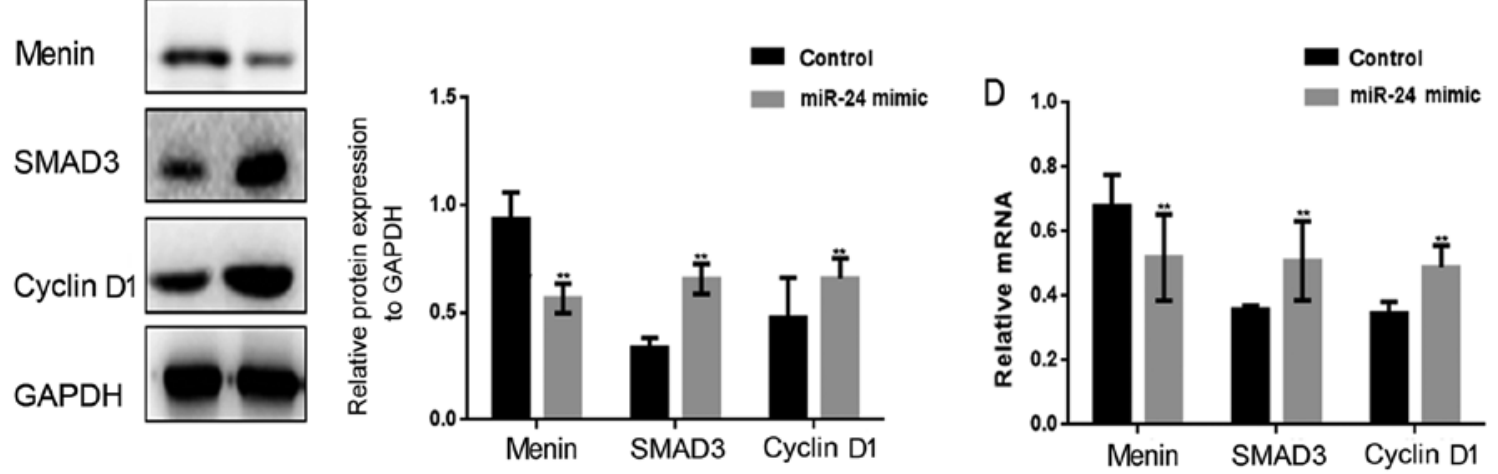

E
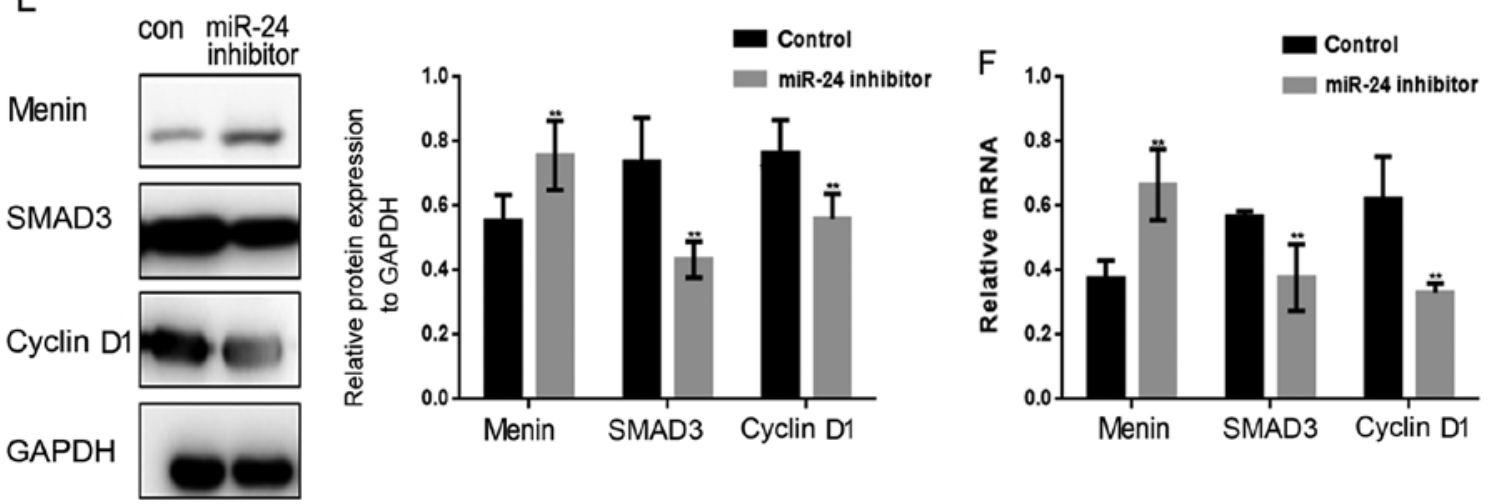

Figure 2. miR-24 promotes the proliferation of lung cancer cells. (A and B) Following transfection with miR-24 mimic/inhibitor, A549 and NCI-H446 cell proliferation was detected by the MTT assay. Data are shown as the mean \pm standard error of the mean (SEM). (C-F) Following transfection of miR-24 mimic/inhibitor in A549 cells, the protein and mRNA levels were detected by western blotting and quantitative polymerase chain reaction analysis. Data are shown as the mean \pm SEM. ${ }^{* *} \mathrm{P}<0.01$.

and mRNA levels of menin, SMAD3 and MMP2 were assessed by western blotting and qPCR when the expression of miR-24 was up- or downregulated in A549 cells (Fig. 4E-H). The results indicated that menin was significantly downregulated, whereas SMAD3 and MMP2 were significantly upregulated by miR-24. 
A

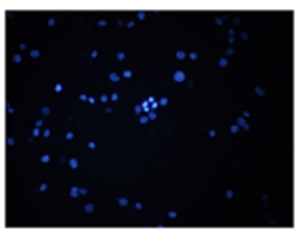

Control

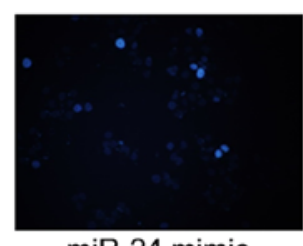

miR-24 mimic
B

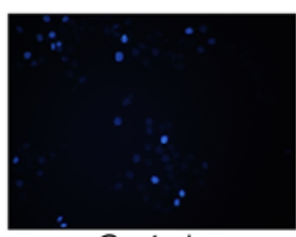

Control

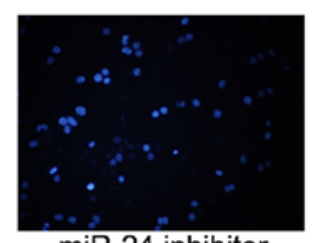

miR-24 inhibitor

C con miR-24

Menin

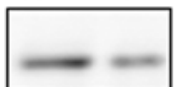

SMAD3

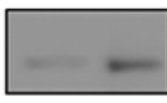

Bcl-2

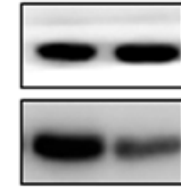

GAPDH
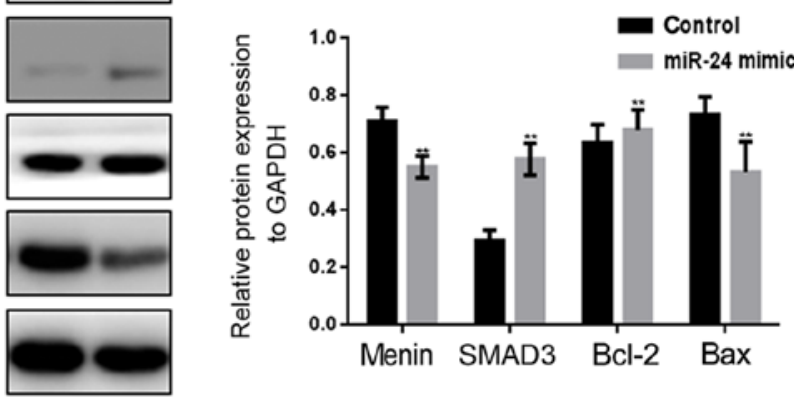

D
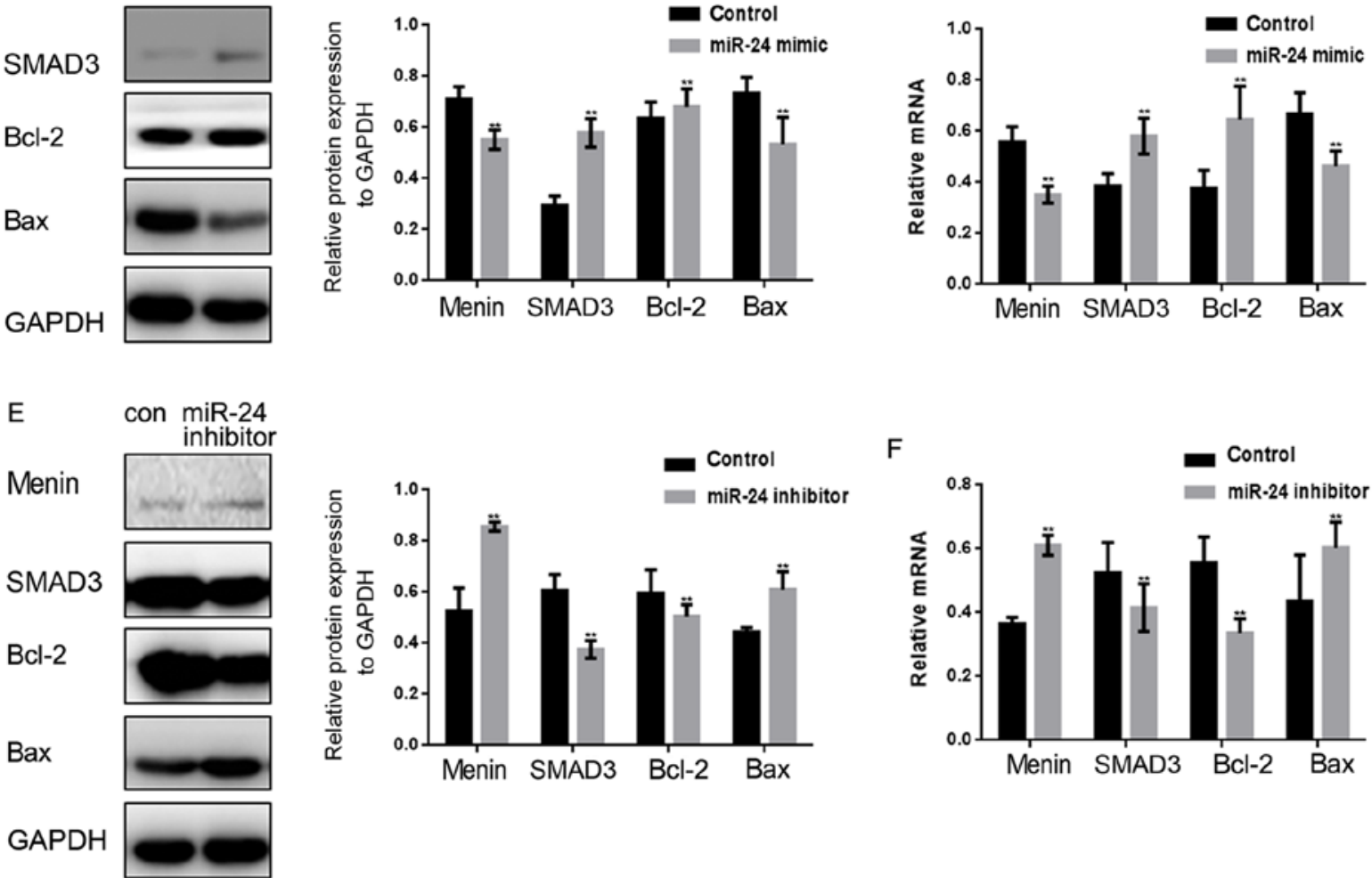

$\mathrm{F}$

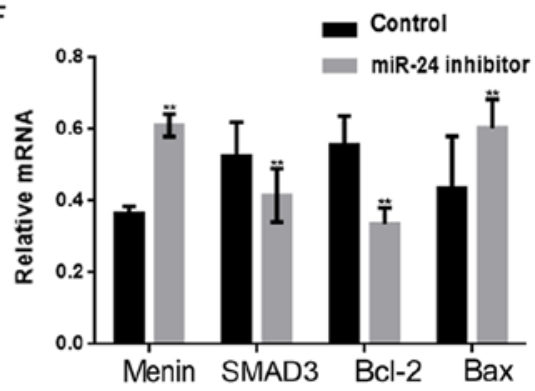

Figure 3. miR-24 inhibits the apoptosis of lung cancer cells. (A and B) Following transfection of miR-24 mimic/inhibitor in A549 cells, cell apoptosis was detected by the Hoechst 33258 assay. (C-F) Following transfection of miR-24 mimic/inhibitor in A549 cells, the protein and mRNA levels were detected by western blotting and quantitative polymerase chain reaction analysis. Data are shown as the mean \pm standard error of the mean $(\mathrm{SEM}){ }^{* * *} \mathrm{P}<0.01$.

\section{Discussion}

Despite the continuous development of early diagnostic and treatment methods for lung cancer, the 5-year survival rate of lung cancer remains $<50 \%$ according to the WHO $(22,23)$. Thus, early detection and timely treatment are crucial for lung cancer patients.

miRNAs are endogenous, small, non-coding, regulatory RNAs that have attracted attention in recent years. A number of reports have indicated that the expression of miRNAs is closely associated with cancer development.

miR-24 has been demonstrated to promote the development of several tumors. In hepatocellular carcinoma (HCC), miR-24 functions as an oncogene, at least in part by promoting cell invasion through downregulation of p53 (24), and miR-24-3p plays an important role in the initiation and progression of HCC by targeting metallothionein $1 \mathrm{M}$ (25). It has also been demonstrated that exosomal miR-24-3p is involved in tumor pathogenesis by mediating T-cell suppression via repression of FGF11, and may serve as a potential prognostic biomarker in nasopharyngeal cancer (NPC) (26). Additionally, it was reported that miR-24-3p significantly inhibited N87 cell growth, migration and invasion, and promoted apoptosis (27). Zhao et al reported that miR-24 may serve as a novel potential biomarker for the prognosis of TSCC patients through targeting FBXW7 (28). It was also demonstrated that miR-24 acts as a tumor suppressor in NPC through targeting FSCN1 (29). In lung cancer, miR-24 may promote cell proliferation by targeting NAIF1 (19). Downregulation contributes to VP16-DDP resistance by targeting ATG4A (21) and enhances tumor invasion and metastasis by targeting PTPN9 and PTPRF to promote EGF signaling (30). All these findings indicate that miR-24 may play an important role in the regulation of lung cancer.

Menin regulates cell proliferation, apoptosis, metastasis and gene transcription. Menin mutations have been detected in $35 \%$ of tumors. One of the proteins interacting with menin is $\beta$-catenin, which acts as a transcription factor, and its dysregulation may be associated with the development and progression of several tumors (31). It has also been reported that menin can interact with K-Ras, SMAD3 and TGF- $\beta$ to exert a biological effect. In human studies, menin expression was found to be lower in lung adenocarcinoma samples, and 
A

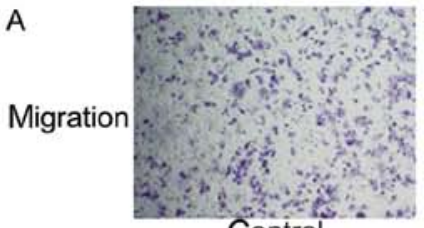

A549

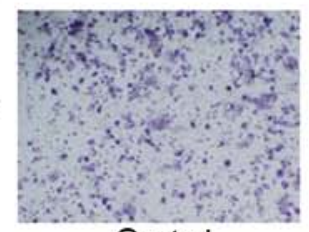

Control

C

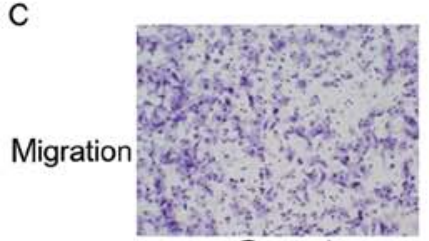

NCl-H446

Invasion

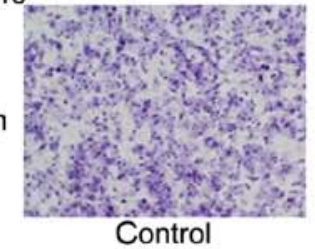

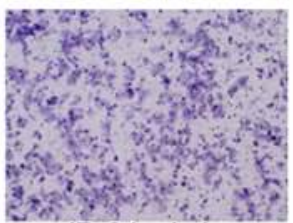

miR-24 mimic

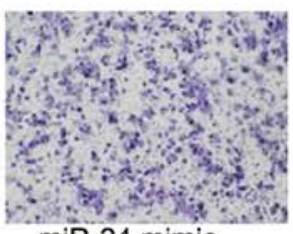

miR-24 mimic

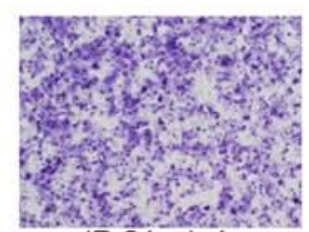

miR-24 mimic

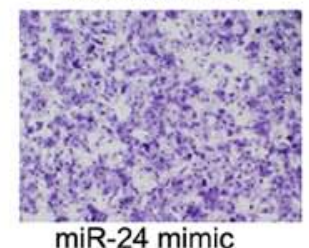

B

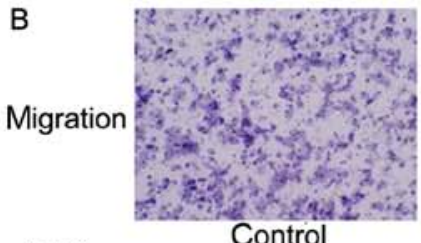

A549

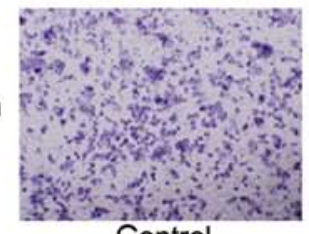

Control

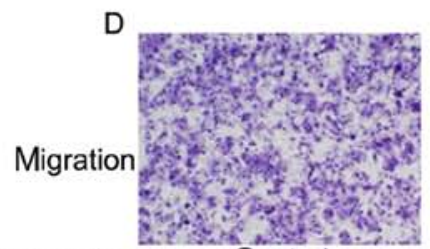

$\mathrm{NCl}-\mathrm{H} 446$

Control

Invasion

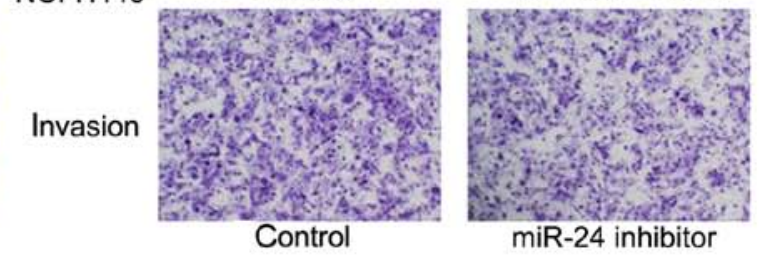

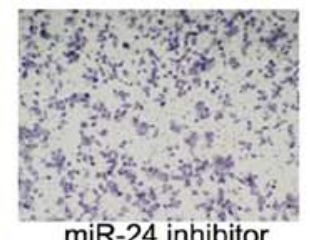

miR-24 inhibitor

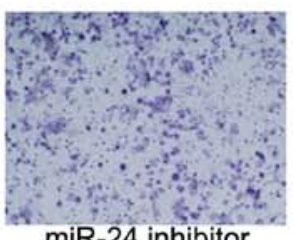

miR-24 inhibitor
E

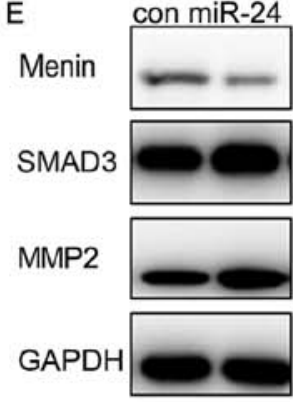

G

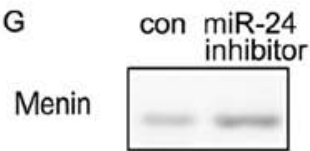

SMAD3

MMP2
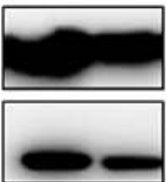

GAPDH

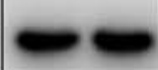

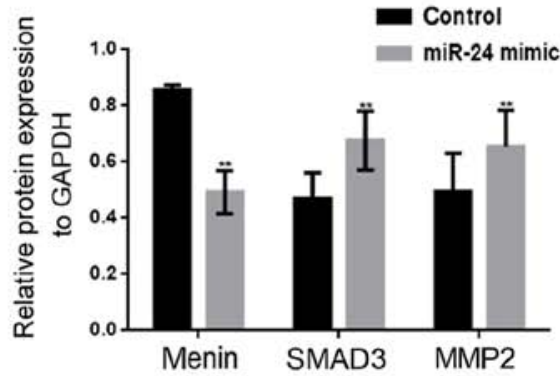

$\mathrm{F}$

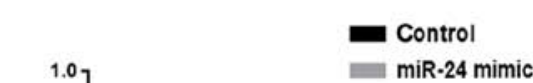

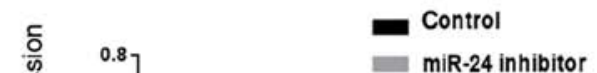

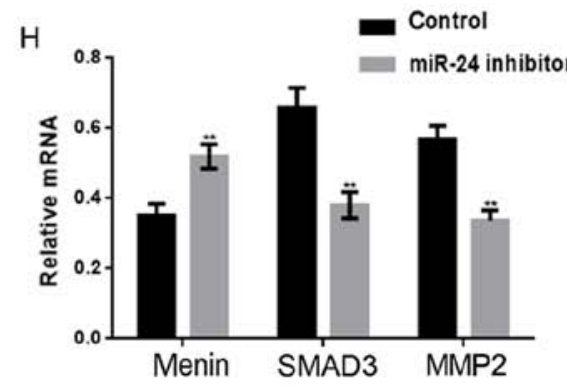

Figure 4. miR-24 promotes the metastasis of lung cancer cells. (A and B) Following transfection of miR-24 mimic/inhibitor in A549 cells, Transwell assay with or without Matrigel was performed. The cells were counted and the results represent the mean \pm standard deviation (SD) of three experiments. ${ }^{* *} \mathrm{P}<0.01$. (C and D) Following transfection of miR-24 mimic/inhibitor in NCI-H446 cells, the Transwell assay with or without Matrigel was performed. The cells were counted and the results represent the mean $\pm \mathrm{SD}$ of three experiments. ${ }^{* *} \mathrm{P}<0.01$. (E-H) Following transfection of miR-24 mimic/inhibitor in A549 cells, the proteins and mRNA levels were detected by western blotting and quantitative polymerase chain reaction analysis. Data are shown as the mean \pm standard error of the mean. ${ }^{* *} \mathrm{P}<0.01$.

it may mediate repression of lung cancer and provide a novel potential target for treating menin-negative and Ras-active lung adenocarcinoma (32).
In our experiments, tumor tissues and adjacent tissues from 70 patients with lung cancer were analyzed. The analysis results revealed that miR-24 expression in the 
tumor tissues was significantly higher compared with that in adjacent tissues, whereas menin expression in the tumor tissues was lower compared with that in the adjacent tissues. In addition, we observed that patients with higher expression of miR-24 had a shorter 5-year survival. By comparison, patients with a higher content of menin survived longer. Thus, it was inferred that miR-24 may be associated with the expression of menin. miR-24 and menin were found to be negatively correlated, and the correlation formula was $\mathrm{y}=-1.014 \mathrm{x}+1.246, \mathrm{R}^{2}=0.4353$. Through the biological software and luciferase reporter assay, it was demonstrated that miR-24 could directly target menin and significantly inhibit its activity, thereby promoting the growth and metastasis of lung cancer cells. The regulatory effect of miR-24 on the growth of lung cancer cells was at least partially realized by targeting menin. The regulatory role of miR-24 in lung cancer was demonstrated through experiments in vitro, and in future experiments our results may be further confirmed in vivo. For the in vivo experiments, a nude mice tumor-bearing model may be constructed, followed by expressing miR-24 in nude mice to detect the proliferation of tumor cells. A mouse lung cancer model may also be constructed to detect the invasion of lung cancer cells overexpressing miR-24.

In summary, we demonstrated that the promotion of the growth and metastasis of lung cancer cells by miR-24 is at least partly mediated by the regulation of menin. Our findings provide a new theoretical basis for the diagnosis and treatment of lung cancer.

\section{Acknowledgements}

Not applicable.

\section{Funding}

The present study was supported by the Key Research Project of Nanjing Military Area Command (grant no. 14ZD32) and the Liaoning Provincial Natural Science Foundation of China (grant no. 201601217).

\section{Availability of data and materials}

The datasets used during the present study are available from the corresponding author upon reasonable request.

\section{Authors' contributions}

YP and HW conceived and designed the study. ZJ, YD, YC and DM performed the experiments. LSH and JHW wrote the paper. LL, FC, FH and YL reviewed and edited the manuscript. All authors read and approved the manuscript and agree to be accountable for all aspects of the research in ensuring that the accuracy or integrity of any part of the work are appropriately investigated and resolved.

\section{Ethics approval and consent to participate}

The study protocols were approved by the Ethics Committee of the General Hospital of Shenyang Military Command. Patient consent was obtained in writing according to institutional regulations.

\section{Consent for publication}

Not applicable.

\section{Competing interests}

The authors declare that they have no competing interests.

\section{References}

1. Lin J, Wang Y, Zou YQ, Chen X, Huang B, Liu J, Xu YM, Li J, Zhang J, Yang WM, et al: Differential miRNA expression in pleural effusions derived from extracellular vesicles of patients with lung cancer, pulmonary tuberculosis, or pneumonia. Tumour Biol Oct 14, 2016.

2. Perepelyuk M, Maher C, Lakshmikuttyamma A and Shoyele SA: Aptamer-hybrid nanoparticle bioconjugate efficiently delivers miRNA-29b to non-small-cell lung cancer cells and inhibits growth by downregulating essential oncoproteins. Int $\mathbf{J}$ Nanomedicine 11: 3533-3544, 2016.

3. Aghanoori MR, Mirzaei B and Tavallaei M: MiRNA molecular profiles in human medical conditions: Connecting lung cancer and lung development phenomena. Asian Pac J Cancer Prev 15: 9557-9565, 2014.

4. Chen Y, Du M, Wang J, Xing P, Zhang Y, Li F and Lu X: MiRNA-200a expression is inverse correlation with hepatocyte growth factor expression in stromal fibroblasts and its high expression predicts a good prognosis in patients with non-small cell lung cancer. Oncotarget 7: 48432-48442, 2016.

5. Zhang $X$, Wang $C$, Shan S, Liu X, Jiang Z and Ren T: TLR4/ROS/miRNA-21 pathway underlies lipopolysaccharide instructed primary tumor outgrowth in lung cancer patients. Oncotarget 7: 42172-42182, 2016.

6. Zhao C, Lu F, Chen H, Zhao F, Zhu Z, Zhao X and Chen H: Clinical significance of circulating miRNA detection in lung cancer. Med Oncol 33: 41, 2016.

7. Zhou Z, Niu X, Li C, Sheng S and Lu S: Inhibition of the growth of non-small cell lung cancer by miRNA-1271. Am J Transl Res 7: 1917-1924, 2015.

8. Bianchi F, Nicassio F, Marzi M, Belloni E, Dall'olio V, Bernard L, Pelosi G, Maisonneuve P, Veronesi G and Di Fiore PP: A serum circulating miRNA diagnostic test to identify asymptomatic high-risk individuals with early stage lung cancer. EMBO Mol Med 3: 495-503, 2011.

9. Chen Y, Min L, Zhang X, Hu S, Wang B, Liu W, Wang R, Gu X, Shen W, Lv H, et al: Decreased miRNA-148a is associated with lymph node metastasis and poor clinical outcomes and functions as a suppressor of tumor metastasis in non-small cell lung cancer. Oncol Rep 30: 1832-1840, 2013.

10. Chen Z, Zeng H, Guo Y, Liu P, Pan H, Deng A and Hu J: miRNA-145 inhibits non-small cell lung cancer cell proliferation by targeting c-Myc. J Exp Clin Cancer Res 29: 151, 2010.

11. Gao W, Lu X, Liu L, Xu J, Feng D and Shu Y: MiRNA-21: A biomarker predictive for platinum-based adjuvant chemotherapy response in patients with non-small cell lung cancer. Cancer Biol Ther 13: 330-340, 2012.

12. Sestini S, Boeri M, Marchianò A, Silva M, Calareso G, Galeone C, Sozzi G and Pastorino U: Lung cancer screening in high-risk subjects: Early detection with LDCT and risk stratification using miRNA-based blood test. Epidemiol Prev 40 (1 Suppl 1): S42-S50, 2016 (In Italian).

13. Feng ZJ, Gao SB, Wu Y, Xu XF, Hua X and Jin GH: Lung cancer cell migration is regulated via repressing growth factor PTN/RPTP $\beta / \zeta$ signaling by menin. Oncogene 29: 5416-5426, 2010.

14. Matkar S, Thiel A and Hua X: Menin: A scaffold protein that controls gene expression and cell signaling. Trends Biochem Sci 38: 394-402, 2013.

15. Duan Y, Hu L, Liu B, Yu B, Li J, Yan M, Yu Y, Li C, Su L, Zhu Z, et al: Tumor suppressor miR-24 restrains gastric cancer progression by downregulating RegIV. Mol Cancer 13: 127, 2014. 
16. Martin EC, Elliott S, Rhodes LV, Antoon JW, Fewell C, Zhu Y, Driver JL, Jodari-Karimi M, Taylor CW, Flemington EK, et al: Preferential star strand biogenesis of pre-miR-24-2 targets PKC-alpha and suppresses cell survival in MCF-7 breast cancer cells. Mol Carcinog 53: 38-48, 2014.

17. Lynch SM, McKenna MM, Walsh CP and McKenna DJ: miR-24 regulates CDKN1B/p27 expression in prostate cancer. Prostate 76: 637-648, 2016.

18. Zhang H, Duan J, Qu Y, Deng T, Liu R, Zhang L, Bai M, Li J, Ning T, Ge S, et al: Onco-miR-24 regulates cell growth and apoptosis by targeting BCL2L11 in gastric cancer. Protein Cell 7 : 141-151, 2016.

19. Zhao G, Liu L, Zhao T, Jin S, Jiang S, Cao S, Han J, Xin Y Dong Q, Liu X and Cui J: Upregulation of miR-24 promotes cell proliferation by targeting NAIF1 in non-small cell lung cancer. Tumour Biol 36: 3693-3701, 2015.

20. Gao Y, Liu Y, Du L, Li J, Qu A, Zhang X, Wang L and Wang C: Down-regulation of miR-24-3p in colorectal cancer is associated with malignant behavior. Med Oncol 32: 362, 2015.

21. Pan B, Chen Y, Song H, Xu Y, Wang R and Chen L: Mir-24-3p downregulation contributes to VP16-DDP resistance in small-cell lung cancer by targeting ATG4A. Oncotarget 6: 317-331, 2015.

22. Yoshida T, Hida T and Yatabe Y: Rapid and dramatic response to alectinib in an anaplastic lymphoma kinase rearranged non-small-cell lung cancer patient who is critically ill. Anticancer Drugs 27: 573-575, 2016.

23. Huang ZL, Cao X, Luo RZ, Chen YF, Zhu LC and Wen Z: Analysis of ERCC1, BRCA1, RRM1 and TUBB3 as predictors of prognosis in patients with non-small cell lung cancer who received cisplatin-based adjuvant chemotherapy: A prospective study. Oncol Lett 11: 299-305, 2016.

24. Chen L, Luo L, Chen W, Xu HX, Chen F, Chen LZ, Zeng WT, Chen JS and Huang XH: MicroRNA-24 increases hepatocellular carcinoma cell metastasis and invasion by targeting p53: miR-24 targeted p53. Biomed Pharmacother 84: 1113-1118, 2016.

25. Dong X, Ding W, Ye J, Yan D, Xue F, Xu L, Yin J and Guo W: MiR-24-3p enhances cell growth in hepatocellular carcinoma by targeting metallothionein 1M. Cell Biochem Funct 34: 491-496, 2016.
26. Ye SB, Zhang H, Cai TT, Liu YN, Ni JJ, He J, Peng JY, Chen QY, Mo HY, Jun-Cui, et al: Exosomal miR-24-3p impedes T-cell function by targeting $F G F 11$ and serves as a potential prognostic biomarker for nasopharyngeal carcinoma. J Pathol 240: 329-340, 2016.

27. Li Q, Wang N, Wei H, Li C, Wu J and Yang G: miR-24-3p regulates progression of gastric mucosal lesions and suppresses proliferation and invasiveness of N87 via peroxiredoxin 6. Dig Dis Sci 61: 486-3497, 2016.

28. Zhao J, Hu C, Chi J, Li J, Peng C, Yun X, Li D, Yu Y, Li Y, Gao M and Zheng X: miR-24 promotes the proliferation, migration and invasion in human tongue squamous cell carcinoma by targeting FBXW7. Oncol Rep 36: 1143-1149, 2016.

29. Li YQ, Lu JH, Bao XM, Wang XF, Wu JH and Hong WQ: MiR-24 functions as a tumor suppressor in nasopharyngeal carcinoma through targeting FSCN1. J Exp Clin Cancer Res 34: 130, 2015.

30. Du WW, Fang L, Li M, Yang X, Liang Y, Peng C, Qian W, O'Malley YQ, Askeland RW, Sugg SL, et al: MicroRNA miR-24 enhances tumor invasion and metastasis by targeting PTPN9 and PTPRF to promote EGF signaling. J Cell Sci 126: 1440-1453, 2013.

31. Veschi S, Lattanzio R, Aceto GM, Curia MC, Magnasco S, Angelucci D, Cama A, Piantelli M and Battista P: Alterations of MEN1 and E-cadherin/ $\beta$-catenin complex in sporadic pulmonary carcinoids. Int J Oncol 41: 1221-1228, 2012.

32. Wu Y, Feng ZJ, Gao SB, Matkar S, Xu B, Duan HB, Lin X, Li SH, Hua X and Jin GH: Interplay between menin and K-Ras in regulating lung adenocarcinoma. J Biol Chem 287: 40003-40011, 2012.

33. Livak KJ and Schmittgen TD: Analysis of relative gene expression data using real-time quantitative PCR and the $2^{-\Delta \Delta C_{\mathrm{T}}}$ method. Methods 25: 402-408, 2001.

34. Pfaffl MW: A new mathematical model for relative quantification in real-time RT-PCR. Nucleic Acids Res 29: e45, 2001.

35. Yang TS, Yang XH, Wang XD, Wang YL, Zhou B and Song ZS: MiR-214 regulate gastric cancer cell proliferation, migration and invasion by targeting PTEN. Cancer Cell Int 13: 68, 2013. 